\title{
Mixed Convective Visco-Elastic MHD Flow with Ohmic Heating
}

\author{
Rita Choudhury \\ Department of Mathematics \\ Gauhati University, Guwahati-781 014, Assam, \\ India
}

\author{
Sajal Kumar Das \\ Department of Mathematics \\ Bajali College, Pathsala, Barpeta, \\ Assam, India.
}

\begin{abstract}
An analysis of mixed convective MHD visco-elastic flow with heat and mass transfer from a vertical plate in presence of ohmic heating and viscous dissipation has been presented. The governing equations of the momentum, thermal and concentration fields are solved by perturbation technique. Expressions for velocity, temperature, concentration profiles and non-dimensional skin-friction coefficient are obtained and illustrated graphically to observe the visco-elastic effect on velocity profile and skinfriction coefficient in combination of other flow parameters involved in the solution.
\end{abstract}

\section{General Terms}

Visco-elastic.

\section{Keywords}

MHD, Grashof number, Prandtl number, skin-friction, visco-elastic, Schmidt number, perturbation technique.

\section{INTRODUCTION}

The study of visco-elastic fluid flows with heat and mass transfer over a vertical porous plate in presence of magnetic field has attracted the researchers for its application in various fields like soil physics, aerodynamics and aeronautics, geophysics, designing of underground water energy storage system, engineering sciences, nuclear power reactors, astrophysics, biological system, geothermal and oil reservoir engineering studies and so on. Nowadays, the period of MHD is of great enlargement and differentiation of subject matter. These problems have attracted the interest of many investigators because of their application in liquid metals, electrolytes and ionized gases. The problem of heat and mass transfer along a vertical plate with variable temperature and concentration in the presence of magnetic field has been studied by Elbashbeshy [1]. Hossain and Rees [2] have studied effect of combined heat and mass transfer in natural convection flow from a vertical wavy surface. Chien-Hsin-Chen [3] has analyzed combined heat and mass transfer in MHD free convection from a vertical surface with ohmic heating and viscous dissipation. Hall current on fluid flow with concentration plays an important role in MHD power generation, in various meteorological and astrophysical studies and in plasma flow through magnetohydrodynamic power generators. Keeping in view of all these facts, a number of researchers have studied the Hall current effect on free and forced convective flows. Acharya et al. [4], Aboeldahab and Elbarbary [5], Datta and Jana [6] have studied the effect of Hall current on MHD flow with heat and mass transfer. Hossain et al. [7] have investigated the effect of radiation on free convection from a porous vertical plate. The radiation effect on MHD free convection flow of a gas at a stretching surface with a uniform free stream has been analyzed by Ghaly and
Elbarbary [8]. Muthukumarswamy and Senthil [9] have studied the effects of heat and mass transfer on moving vertical plate in the presence of thermal radiation. In all the above investigations, the combined effect of ohmic heating and viscous dissipation are not analyzed. Hossain [10] has studied the effects of viscous and Joule heating on free convective MHD flow with variable plate temperature. Babu and Reddy [11] have analyzed the mass transfer effects on MHD mixed convective flow from a vertical surface with ohmic heating and viscous dissipation Ganesan and Palani [12] have investigated the numerical solution of unsteady MHD flow past a semi-infinite isothermal vertical plate. Ganesan and Palani [13] have also presented a finite difference analysis of unsteady natural convection MHD flow past an inclined plate with variable surface heat and mass flux. Aydin and Kaya [14] have analyzed the non-Darcian forced convection flow of viscous dissipating fluid over a flat plate embedded in a porous medium. Aydin and Kaya [15] have also studied MHD mixed convection of a viscous dissipating fluid about permeable vertical flat plate.

In all the above cases, the fluid models have been considered as Newtonian. But the mechanisms of nonNewtonian fluid flows are used in various manufacturing processes and hence play an important role in modern technology and industrial applications. Authors like Kelly et al.[16], Shbhash et al. [17], Sonth et al. [18], Abel et al. [19], Choudhury and Mahanta [20], Choudhury and Dey [21], Choudhury and Das [22] etc. have analyzed some problems of physical interest in this field. Bachok et al. [23] have studied MHD flow and heat transfer near the stagnation point on a stretching/shrinking sheet in a micropolar fluid.

In this paper, the mixed convective MHD flow with heat and mass transfer from a vertical porous plate in presence of ohmic heating and viscous dissipation has been studied to observe the visco-elastic effects on the fluid flow field along with other flow parameters involved in the problem. The electrically conducting visco-elastic fluid flow is characterized by Walters liquid (Model B').

The constitutive equation for Walters liquid (Model $\mathrm{B}^{\prime}$ ) is

$$
\sigma_{i k}=-p g_{i k}+\sigma_{i k}^{\prime}, \quad \sigma_{i k}^{\prime}=2 \eta_{0} e^{i k}-2 K_{0} e^{\prime i k} \quad \text { (1) }
$$

where $\sigma_{i k}$ is the stress tensor, $p$ is isotropic pressure, $g_{i k}$ is the metric tensor of a fixed co-ordinate system $x^{i}, v^{i}$ is the velocity vector, the contravariant form of $\mathrm{e}^{\text {ik }}$ is given by $e^{\prime i k}=\frac{\partial e^{i k}}{\partial t}+v^{m} e^{i k}{ }_{m}-v^{i}{ }_{m} e^{i m}-v^{i}{ }_{m} e^{m k}$

It is the convected derivative of the deformation rate tensor $\mathrm{e}^{\mathrm{ik}}$ defined by

$2 \mathrm{e}_{\mathrm{ik}}=\mathrm{v}_{\mathrm{i}, \mathrm{k}}+\mathrm{v}_{\mathrm{k}, \mathrm{i}}$ 
Here $\eta_{0}$ is the limiting viscosity at the small rate of shear which is given by

$\eta_{0}=\int_{0}^{\infty} N(\tau) d \tau$ and $k_{0}=\int_{0}^{\infty} \tau N(\tau) d \tau$

$\mathrm{N}(\tau)$ being the relaxation spectrum as introduced by Walters $[24,25]$. This idealized model is a valid approximation of Walters liquid (Model $\mathrm{B}^{\prime}$ ) taking very short memories into account so that terms involving

$\int_{0}^{\infty} \tau^{n} N(\tau) d \tau, \quad n \geq 2$

have been neglected.

\section{MATHEMATICAL FORMULATION}

The mixed convective MHD flow with heat and mass transfer over a vertical plate in presence of ohmic heating has been considered. The $\bar{x}$-axis is taken along the length of the non-conducting porous plate and $\bar{y}$-axis is perpendicular to it. Let $\bar{u}$ and $\bar{v}$ be the velocities of the fluid along $\bar{x}$ and $\bar{y}$ directions respectively. As the motion is two dimensional and the plate is long enough, all the physical quantities are independent of $\bar{x}$. The equations governing the fluid flow are:

equation of continuity:

$$
\frac{\partial \bar{v}}{\partial \bar{y}}=0
$$

$\Rightarrow \bar{v}=-v_{0}$ (constant)

also $\frac{\partial \bar{P}}{\partial \bar{y}}=0$

$\Rightarrow \bar{P}$ is independent of $\bar{y}$.

momentum equation:

$$
\begin{aligned}
\bar{v} \frac{\partial \bar{u}}{\partial \bar{y}}= & v \frac{\partial^{2} \bar{u}}{\partial \bar{y}^{2}}-\frac{K_{0}}{\rho} \bar{v} \frac{\partial^{3} \bar{u}}{\partial \bar{t} \partial \bar{y}^{2}}+g \beta\left(\bar{T}-\bar{T}_{\infty}\right)-\frac{\sigma B_{0}{ }^{2} \bar{u}}{\rho}+ \\
& g \bar{\beta}\left(\bar{C}-\bar{C}_{\infty}\right)-\frac{v}{\bar{K}} \bar{u}
\end{aligned}
$$

energy equation:

$$
\begin{aligned}
\bar{v} \frac{\partial \bar{T}}{\partial \bar{y}}= & \frac{\kappa}{\rho C_{p}} \frac{\partial^{2} \bar{T}}{\partial \bar{y}^{2}}+\frac{v}{C_{p}}\left(\frac{\partial \bar{u}}{\partial \bar{y}}\right)^{2}-K_{0}\left(\bar{v} \frac{\partial \bar{u}}{\partial \bar{y}} \frac{\partial^{2} \bar{u}}{\partial \bar{y}^{2}}\right)+\frac{\sigma B_{0}{ }^{2} \bar{u}^{2}}{\rho C_{p}}+ \\
& Q\left(\bar{T}_{\infty}-\bar{T}\right)-\frac{1}{\rho C_{p}} \frac{\partial q_{r}}{\partial \bar{y}}
\end{aligned}
$$

concentration equation:

$\bar{v} \frac{\partial \bar{C}}{\partial \bar{y}}=D \frac{\partial^{2} \bar{C}}{\partial \bar{y}^{2}}$

where $v$ is the kinematic viscosity, $g$ is the acceleration due to gravity, $\beta$ is the volumetric co-efficient of expansion for heat transfer, $\bar{\beta}$ is the volumetric co-efficient of expansion for the fluid, $\rho$ is the density, $\bar{T}$ is the temperature of the fluid near the plate, $\bar{T}_{\infty}$ is the free stream temperature of the fluid, $\kappa$ is the thermal conductivity, $C_{p}$ is the specific heat at constant temperature, $C_{s}$ is the concentration susceptibility, $\bar{C}$ is the mass concentration, $q_{r}$ is the radiative heat flux, $B_{0}$ is the magnetic field, $\mathrm{D}$ is the molecular diffusivity, $v_{0}$ is the constant suction velocity, $\sigma$ is the magnetic permeability, $\bar{K}$ is the permeability of the porous medium and $\bar{P}$ is the pressure.

The radiative heat flux $q_{r}$ [26] is given by

$\frac{\partial q_{r}}{\partial \bar{y}}=4\left(\bar{T}-T_{\infty}\right) I$

where $I=\int_{0}^{\infty} K_{\lambda w} \frac{\partial e_{b \lambda}}{\partial \bar{T}} d \lambda, K_{\lambda w}$ is the absorption coefficient at the wall and $\mathrm{e}_{\mathrm{b} \lambda}$ is Planck's function.

The initial boundary conditions are

$\bar{y}=0: \bar{u}=0, \bar{T}=T_{w}, \bar{C}=C_{w}$

$\bar{y} \rightarrow \infty: \bar{u} \rightarrow 0, \bar{T} \rightarrow T_{\infty}, \bar{C} \rightarrow C_{\infty}$

On introduction of the non-dimensional quantities $y=\frac{v_{0} \bar{y}}{v}, u=\frac{\bar{u}}{v_{0}}, T=\frac{\bar{T}-\bar{T}_{\infty}}{T_{w}-T_{\infty}}, C=\frac{\bar{C}-\bar{C}_{\infty}}{C_{w}-C_{\infty}}$,

$$
\begin{aligned}
& P_{r}=\frac{\mu C_{p}}{\kappa}, \quad S_{C}=\frac{v}{D}, M=\frac{\sigma B_{0}^{2} v}{\rho v_{0}^{2}}, G_{r}=\frac{\rho g \beta v\left(T_{w}-T_{\infty}\right)}{v_{0}{ }^{3}}, \\
& G_{m}=\frac{\rho g \beta v\left(C_{w}-C_{\infty}\right)}{v_{0}^{3}}, K=\frac{\bar{K} v_{0}^{2}}{v}, E_{C}=\frac{v_{0}^{2}}{C_{p}\left(C_{w}-C_{\infty}\right)}, \\
& N=\frac{4 v I}{\rho C_{p} v_{0}^{2}}, H=\frac{Q v}{\rho C_{p} V_{0}^{2}} .
\end{aligned}
$$

equations (7), (8) and (9) become

$K_{1} \frac{\partial^{3} y}{\partial y^{3}}+\frac{\partial^{2} y}{\partial y^{2}}+\frac{\partial u}{\partial y}-\left(M+\frac{1}{K}\right) u=-G_{r} T-G_{m} C$

$\frac{\partial^{2} T}{\partial y^{2}}+P_{r} \frac{\partial T}{\partial y}-P_{r}(N+H) T=-P_{r} E_{c}\left(\frac{\partial u}{\partial y}\right)^{2}-P_{r} M E_{c} u^{2}-$

$$
K_{1} P_{r} E_{c} \frac{\partial u}{\partial y} \frac{\partial^{2} y}{\partial y^{2}}
$$

$\frac{\partial^{2} C}{\partial y^{2}}+S_{C} \frac{\partial C}{\partial y}=0$

where $K_{1=}=\frac{K_{0} v_{0}^{2}}{\rho v^{2}}, G_{r}$ is the Grashof number for heat transfer, $G_{m}$ is the Grashof number for mass transfer, $P_{r}$ is the Prandtl number, $N$ is the radiation parameter, $S_{c}$ is the Schmidt number, $E_{c}$ is the Eckert number, $M$ is the magnetic parameter and $H$ is the heat source parameter.

The modified boundary conditions are:

$y=0: u=0, T=1, C=1$

$y \rightarrow \infty: u \rightarrow 0, T \rightarrow 0, C \rightarrow 0$

\section{METHOD OF SOLUTION}

To solve the equations (12) to (14), the perturbation scheme for $E_{c} \ll 1$ is introduced as follows:

$$
\begin{aligned}
& u(y)=u_{0}(y)+E_{c} u_{1}(y)+o\left({E_{c}}^{2}\right) \\
& T(y)=T_{0}(y)+E_{c} T_{1}(y)+o\left({E_{c}}^{2}\right) \\
& C(y)=C_{0}(y)+E_{c} C_{1}(y)+o\left({E_{c}}^{2}\right)
\end{aligned}
$$

On substituting relations (16) in equations (12) to (14) and neglecting the second and higher powers of $E_{c}$, the zeroth and first order equations are obtained as follows: zeroth order equations:

$$
\begin{aligned}
& K_{1} \frac{d^{3} u_{0}}{d y^{3}}+\frac{d^{2} u_{0}}{d y^{2}}+\frac{d u_{0}}{d y}-\left(M+\frac{1}{K}\right) u_{0}=-G_{r} T_{0}-G_{m} C_{0} \\
& \frac{\partial^{2} T_{0}}{\partial y^{2}}+P_{r} \frac{\partial T_{0}}{\partial y}-P_{r}(N+H) T_{0}=0 \\
& \frac{\partial^{2} C_{0}}{\partial y^{2}}+S_{c} \frac{\partial C_{0}}{\partial y}=0
\end{aligned}
$$

first order equations:

$$
\begin{aligned}
& K_{1} \frac{d^{3} u_{1}}{d y^{3}}+\frac{d^{2} u_{1}}{d y^{2}}+\frac{d u_{1}}{d y}-\left(M+\frac{1}{K}\right) u_{1}=-G_{r} T_{1}-G_{m} C_{1} \\
& \frac{\partial^{2} T_{1}}{\partial y^{2}}+P_{r} \frac{\partial T_{1}}{\partial y}-P_{r}(N+H) T_{1}=-P_{r}\left(\frac{d u_{0}}{d y}\right)^{2}-P_{r} M u_{0}^{2}- \\
& K_{1} P_{r} \frac{d u_{0}}{d y} \frac{d^{2} u_{0}}{d y^{2}}
\end{aligned}
$$

The new boundary conditions are now, $y=0: u_{0}=0, u_{1}=0, T_{0}=1, T_{1}=0, C_{0}=1, C_{1}=0$ $y \rightarrow \infty: u_{0} \rightarrow 0, u_{1} \rightarrow 0, T_{0} \rightarrow 0, T_{1} \rightarrow 0, C_{0} \rightarrow 0, C_{1} \rightarrow 0$

In order to solve the equations (17) and (20), the multi-perturbation scheme for $K_{1} \ll 1$ (for low shear stress) has been introduced as follows:

$u_{0}=u_{00}+K_{1} u_{01}+o\left(K_{1}^{2}\right)$
$u_{1}=u_{10}+K_{1} u_{11}+o\left(K_{1}^{2}\right)$

Using relations (24) in (17) and (20) and neglecting the second and higher powers of $K_{1}$, the zeroth and first order equations are obtained as

$\frac{d^{2} u_{00}}{d y^{2}}+\frac{d u_{00}}{d y}-\left(M+\frac{1}{K}\right) u_{00}=-G_{r} T_{0}-G_{m} C_{0}$ 
$\frac{d^{2} u_{10}}{d y^{2}}+\frac{d u_{10}}{d y}-\left(M+\frac{1}{K}\right) u_{10}=-G_{r} T_{1}-G_{m} C_{1}$

and

$\frac{d^{2} u_{01}}{d y^{2}}+\frac{d u_{01}}{d y}-\left(M+\frac{1}{K}\right) u_{01}=-\frac{d^{3} u_{00}}{d y^{3}}$

$\frac{d^{2} u_{11}}{d y^{2}}+\frac{d u_{11}}{d y}-\left(M+\frac{1}{K}\right) u_{11}=-\frac{d^{3} u_{10}}{d y^{3}}$

The corresponding boundary conditions are $y=0: u_{00}=0, u_{01}=0, u_{10}=0, u_{11}=0$

$y \rightarrow \infty: u_{00} \rightarrow 0, u_{01} \rightarrow 0, u_{10} \rightarrow 0, u_{11} \rightarrow 0$

The solutions of equations (19), (22), (18), (21), and (25) to (28) subject to boundary conditions (23) and (29) are obtained as follows:

$$
\begin{aligned}
C_{0}= & e^{-S_{c} y} \\
C_{1}= & 0 \\
T_{0}= & e^{-q_{1} y} \\
T_{1}= & b_{40} e^{-q_{1} y}+b_{30} e^{-2 q_{1} y}+b_{31} e^{-2 q_{2} y}+b_{32} e^{-2 S_{c} y}+ \\
& b_{33} e^{-\left(q_{1}+q_{2}\right) y}+b_{34} e^{-\left(q_{1}+S_{c}\right) y}+b_{35} e^{-\left(q_{2}+S_{c}\right) y}+ \\
& b_{36} y e^{-2 q_{2} y}+b_{37} y e^{-\left(q_{1}+q_{2}\right) y}+b_{38} y e^{-\left(q_{2}+S_{c}\right) y}+ \\
& b_{39} y^{2} e^{-2 q_{2} y} \\
u_{00}= & b_{1} e^{-q_{2} y}+b_{2} e^{-q_{1} y}+b_{3} e^{-S_{c} y} \\
u_{01}= & b_{4} e^{-q_{2} y}+b_{5} y e^{-q_{2} y}+b_{6} e^{-q_{1} y}+b_{7} e^{-s_{c} y} \\
u_{10}= & b_{52} e^{-q_{2} y}+b_{41} e^{-q_{1} y}+b_{42} e^{-2 q_{1} y}+b_{43} e^{-2 q_{2} y}+ \\
& b_{44} e^{-2 S_{c} y}+b_{45} e^{-\left(q_{1}+q_{2}\right) y}+b_{46} e^{-\left(q_{1}+S_{c}\right) y}+ \\
& b_{47} e^{-\left(q_{2}+S_{c}\right) y}+b_{48} y e^{-2 q_{2} y}+b_{49} y e^{-\left(q_{1}+q_{2}\right) y}+ \\
& b_{50} y e^{-\left(q_{2}+S_{c}\right) y}+b_{51} y^{2} e^{-2 q_{2} y} \\
u_{11}= & b_{65} e^{-q_{2} y}+b_{53} e^{-q_{1} y}+b_{54} y e^{-q_{2} y}+b_{55} e^{-2 q_{1} y}+ \\
& b_{56} e^{-2 q_{2} y}+b_{57} e^{-2 S_{c} y}+b_{58} e^{-\left(q_{1}+q_{2}\right) y}+ \\
& b_{59} e^{-\left(q_{1}+S_{c}\right) y}+b_{60} e^{-\left(q_{2}+S_{c}\right) y}+b_{61} y e^{-2 q_{2} y}+ \\
& b_{62} y e^{-\left(q_{1}+q_{2}\right) y}+b_{63} y e^{-\left(q_{2}+S_{c}\right) y}+b_{64} y^{2} e^{-2 q_{2} y}
\end{aligned}
$$

The constants are obtained but not given here due to brevity.

\section{RESULTS AND DISCUSSION}

The expressions for velocity, temperature and concentration for the flow field are given by

$u=u_{00}+K_{1} u_{01}+E_{c}\left(u_{10}+K_{1} u_{11}\right)$

$T=T_{0}+E_{c} T_{1}$

$C=C_{0}+E_{c} C_{1}$

The non-dimensional skin friction at the wall of the plate is given by

$$
\begin{gathered}
\sigma_{0}=\left(u_{00}{ }^{\prime}+K_{1} u_{01}{ }^{\prime}+K_{1}\left(u_{00}{ }^{\prime \prime}+K_{1} u_{01}{ }^{\prime \prime}\right)+E_{c}\left[u_{10}{ }^{\prime}+\right.\right. \\
\left.\left.K_{1} u_{11}{ }^{\prime}+K_{1}\left(u_{10}{ }^{\prime}+K_{1} u_{11^{\prime \prime}}\right)\right]\right)_{y=0}
\end{gathered}
$$

The non-dimensional form of the rate of heat transfer in the form of Nusselt number $\mathrm{N}_{\mathrm{u}}$ is given by,

$$
N_{u}=\left(\frac{\partial T}{\partial y}\right)_{y=0}=\left(T_{0}{ }^{\prime}+E_{c} T_{1}^{\prime}\right)_{y=0}
$$

The non-dimensional form of the rate of mass transfer at the plate in terms of Sherwood number $S_{h}$ is given by

$$
S_{h}=\left(\frac{\partial C}{\partial y}\right)_{y=0}=\left(C_{0}{ }^{\prime}+E_{c} C_{1}{ }^{\prime}\right)_{y=0}
$$

\subsection{Discussions}

The objective of this study is to find out the effects of visco-elastic parameter on mixed convective MHD flow with heat and mass transfer from a vertical plate in presence of ohmic heating and viscous dissipation as the effects of other flow parameters have been analyzed by Babu and Reddy[11]. The non zero values of the non-dimensional parameter $K_{I}$ reveals the visco-elastic effects while $K_{I}=0$ represents those for Newtonian fluid. The expressions for the velocity field $u$, the temperature field $T$ and the mass transfer $C$ may be obtained from (38), (39) and (40) respectively.

The numerical calculations are to be carried out for Prandtl number $P_{r}=3$, Eckert number $E_{c}=.001$, permeability parameter $K=1$, radiation parameter $N=5$, Schmidt number $S_{c}=.4$ in all the cases. The profiles of velocity component $u$ against $y$ are depicted for various flow parameters involved in the problem in the figures 1 to 4 to observe the viscoelastic effect in presence of magnetic parameter $M$ and heat source parameter $H(M=.5, H=3)$.It is visualized from these figures that the velocity accelerates and then decelerates in both Newtonian and non- Newtonian cases. Again, an accelerated trend is noticed during the modification of visco-elasticity in comparison with Newtonian fluid. Also, in our study $G_{r}$ and $G_{m}$ characterize the free convection parameter for heat transfer and mass transfer respectively.

The figures 1 and 2 depict that the magnitude of the maximum value of velocity is less for externally heated plate $\left(G_{r}<0\right.$ than that for externally cooled plate $\left(G_{r}>0\right.$.

The magnitude of the maximum value of velocity is less when $G_{r}<G_{m}$ in comparison with $G_{r}=G_{m}$ (figures1, 3).

Again, the magnitude of the maximum value of velocity is diminished remarkably when $G_{r}>G_{m}$ as compared to $G_{r}=G_{m}$ (figures 1,4$)$.

Figures 5 to 8 and 9 to 12 illustrate the behavior of skin friction coefficient $\sigma_{0}$ against the heat source parameter $H$ and the magnetic parameter $M$ respectively.

The skin friction coefficient diminishes (figure 5) with the increasing value of $H$ for externally cooled plate $\left(G_{r}>0\right)$ but enhances (figure 6) for externally heated plate $\left(\mathrm{G}_{\mathrm{r}}<0\right)$ along with the increasing values of the visco-elastic parameter $K_{I}$

For $G_{r} \neq G_{m}$, the skin friction coefficient against $H$ exhibits the same behavior as that in externally cooled plate for $G_{r}=G_{m}$ (figures 7,8). But the magnitude of the skin friction coefficient diminishes in the case of $G_{r} \neq G_{m}$ as compared to $G_{r}=G_{m}$ in both Newtonian and nonNewtonian cases.

Figure 9 reveals a diminishing trend of the skin friction coefficient with the enhancement of the visco-elastic parameter $K_{I}$ as well as magnetic parameter $M$ for externally cooled plate $\left(\mathrm{G}_{\mathrm{r}}>0\right)$ but the figure 10 exhibits an opposite trend for externally heated plate $\left(\mathrm{G}_{\mathrm{r}}<0\right)$.

Also, for the growth of the visco-elastic parameter $K_{l}$ and the magnetic parameter $M$, the shearing stress $\sigma_{0}$ experiences a declination for externally cooled plate $\left(\mathrm{G}_{\mathrm{r}}>0\right)$ when $G_{r} \neq G_{m}$ (figures 11,12 ).

It is also found the from figures 9,11 and 12 that the magnitude of the skin friction diminishes for $G_{r} \neq G_{m}$ as compared to $G_{r}=G_{m}$ with a higher variation in the case of $G_{r}>G_{m}$ than in $G_{r}<G_{m}$.

It is noticed that the thermal and concentration fields are affected by the magnetic parameter with the combination of other flow parameters in Newtonian case but they are not significantly affected by the visco-elastic parameter. 


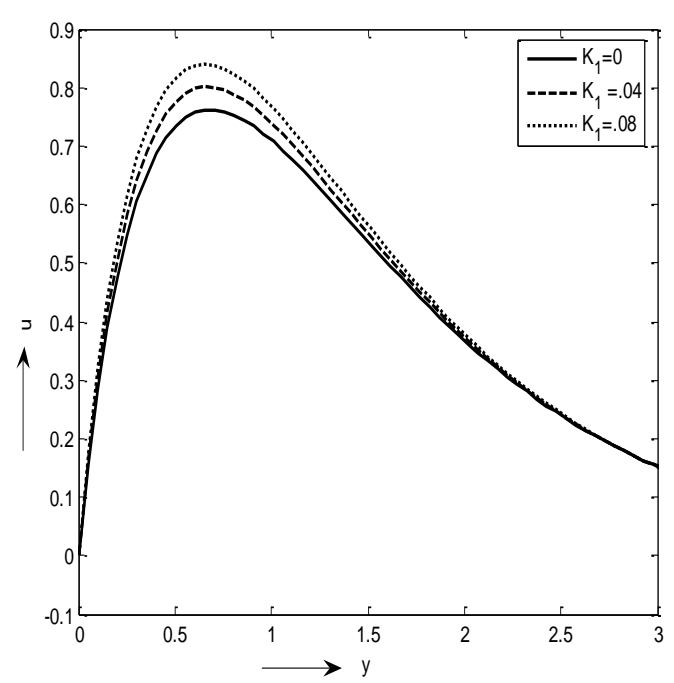

Figure 1: Velocity profile $u$ against $y$ for $G_{r}=5, G_{m}=5$

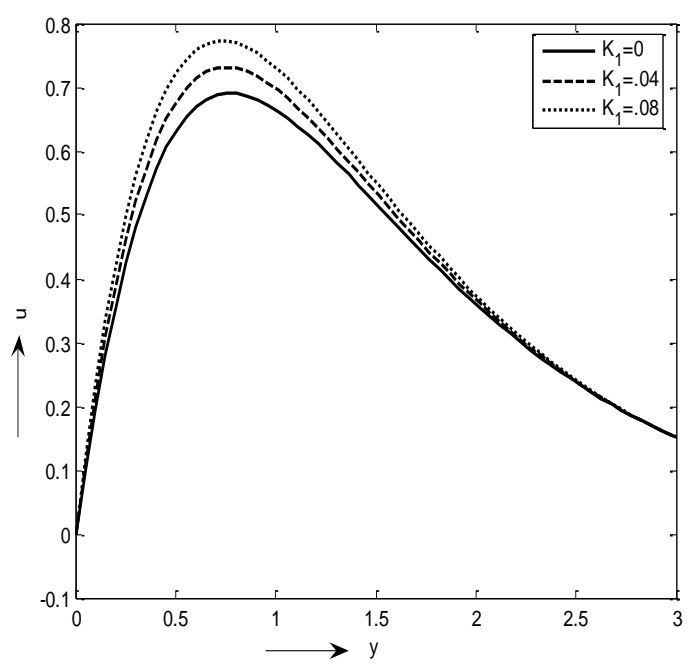

Figure 2: Velocity profile $u$ against $y$ for $G_{r}=-5, G_{m}=5$

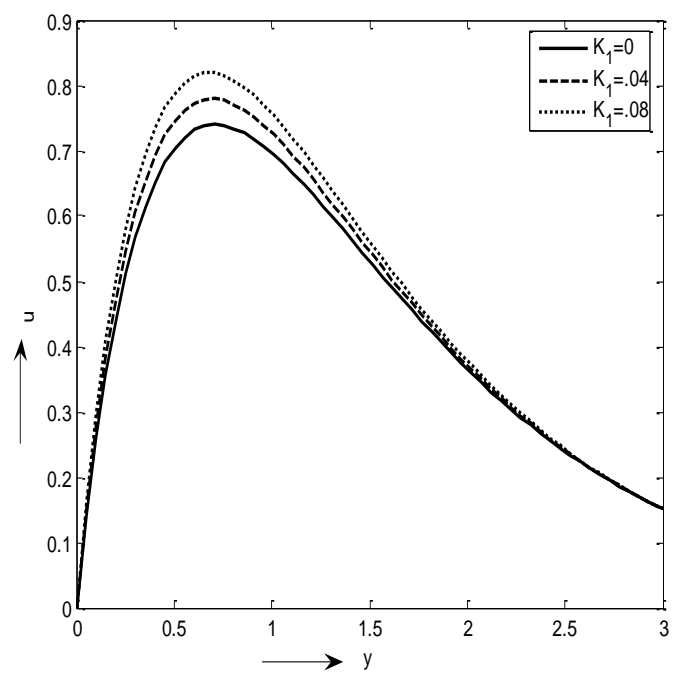

Figure 3: Velocity profile $u$ against $y$ for $G_{r}=2, G_{m}=5$

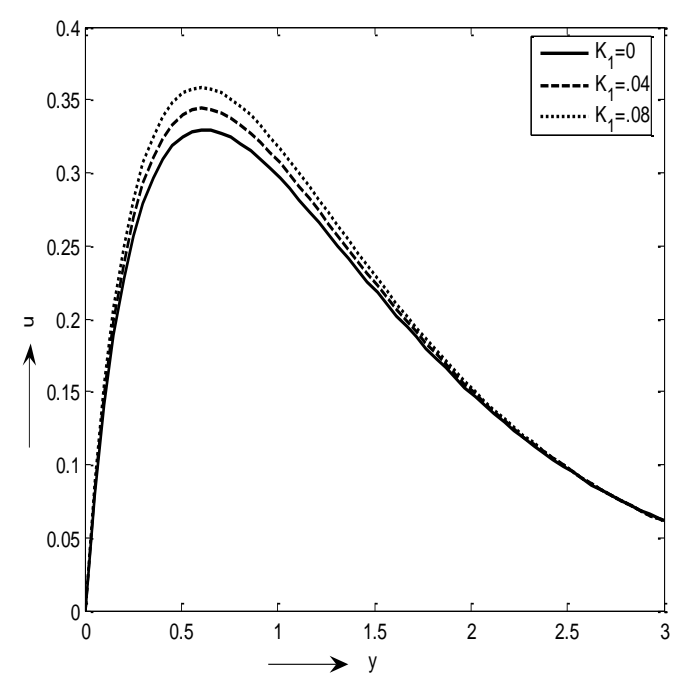

Figure 4: Velocity profile $u$ against $y$ for $G_{r}=5, G_{m}=2$

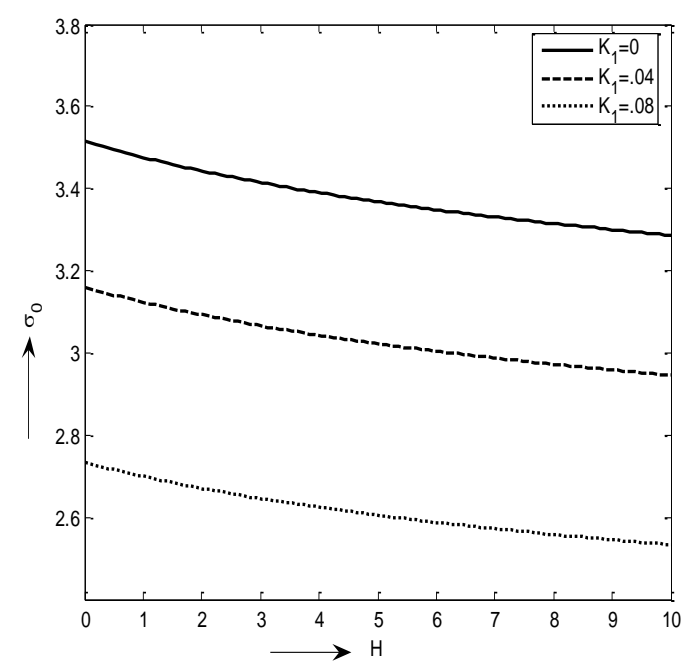

Figure 5: Skin-friction coefficient $\sigma_{0}$ against $H$ for $G_{r}=5$, $G_{m}=5, M=.5$

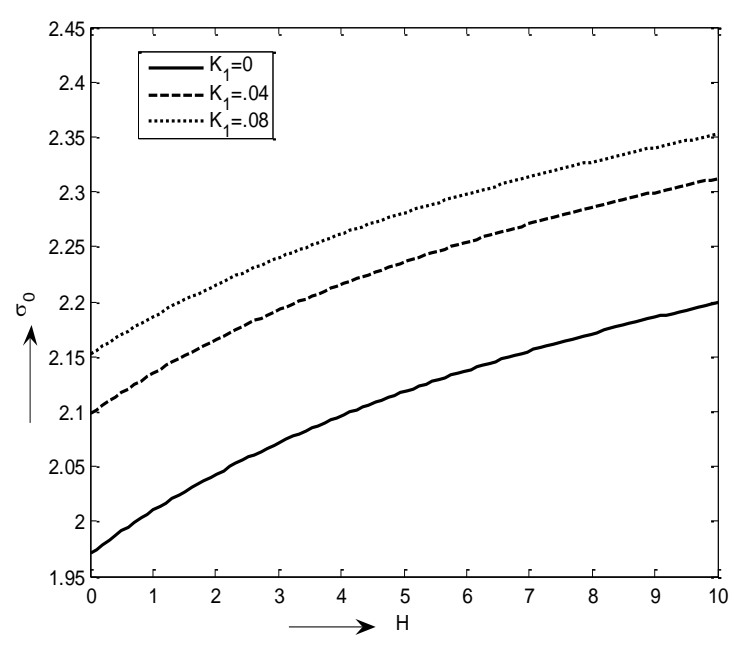

Figure 6: Skin-friction coefficient $\sigma_{0}$ against $H$ for $G_{r}=-5, G_{m}=5, M=.5$ 


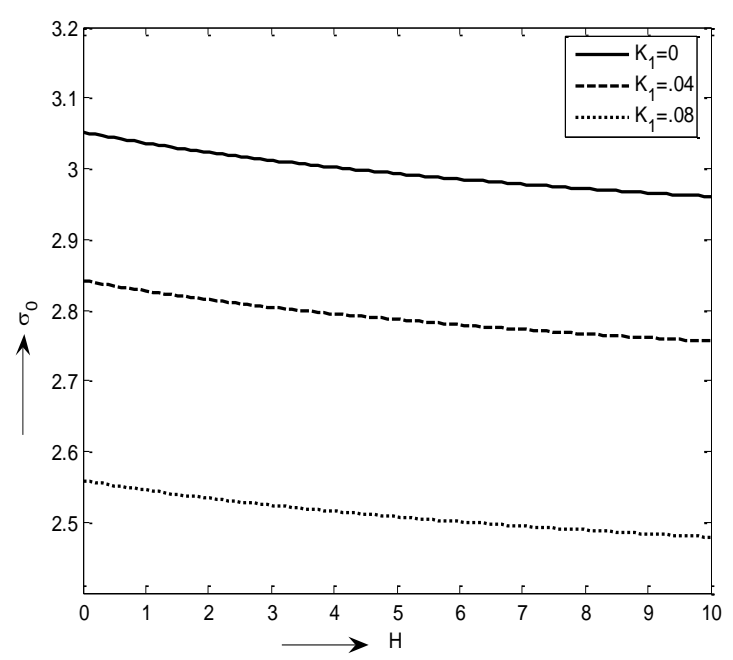

Figure 7: Skin-friction coefficient $\sigma_{0}$ against $H$ for $G_{r}=2, G_{m}=5, M=.5$

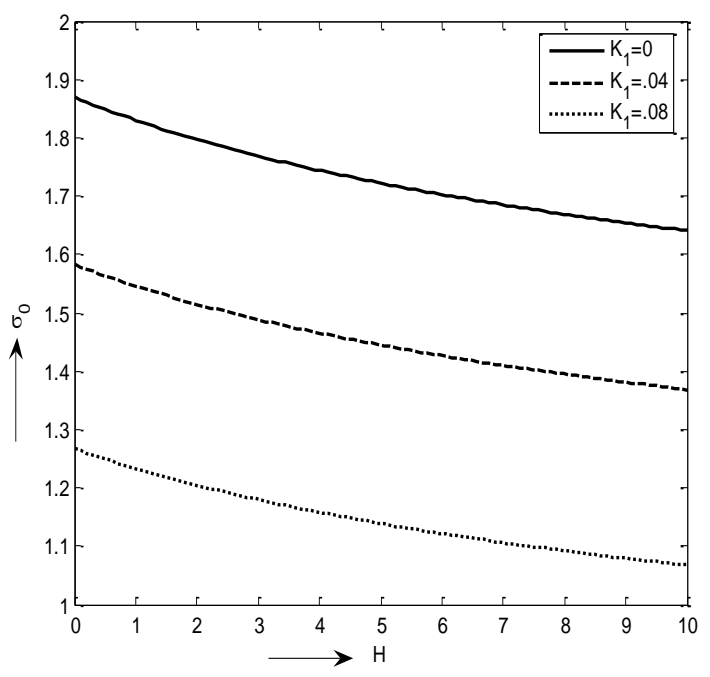

Figure 8: Skin-friction coefficient $\sigma_{0}$ against $H$ for $G_{r}=5, G_{m}=2, M=.5$

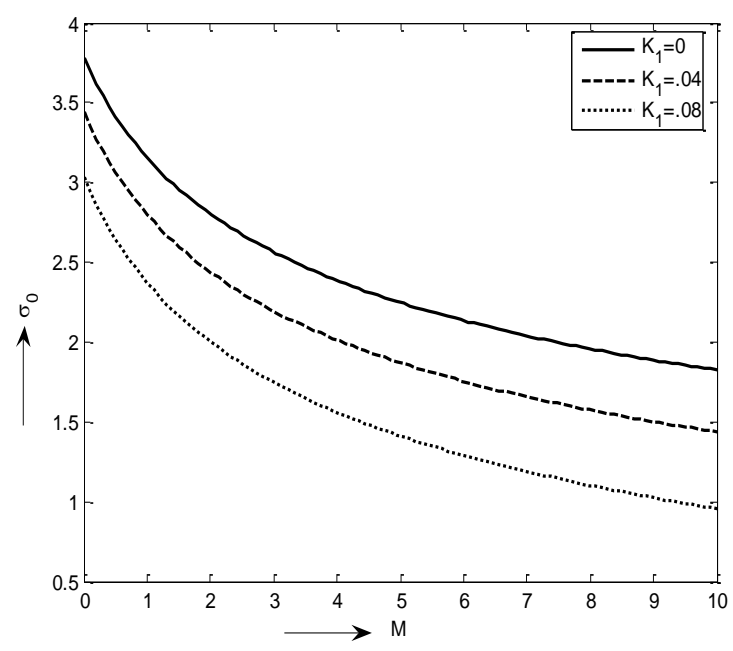

Figure 9: Skin-friction coefficient $\sigma_{0}$ against $M$ for $G_{r}=5, G_{m}=5, H=3$

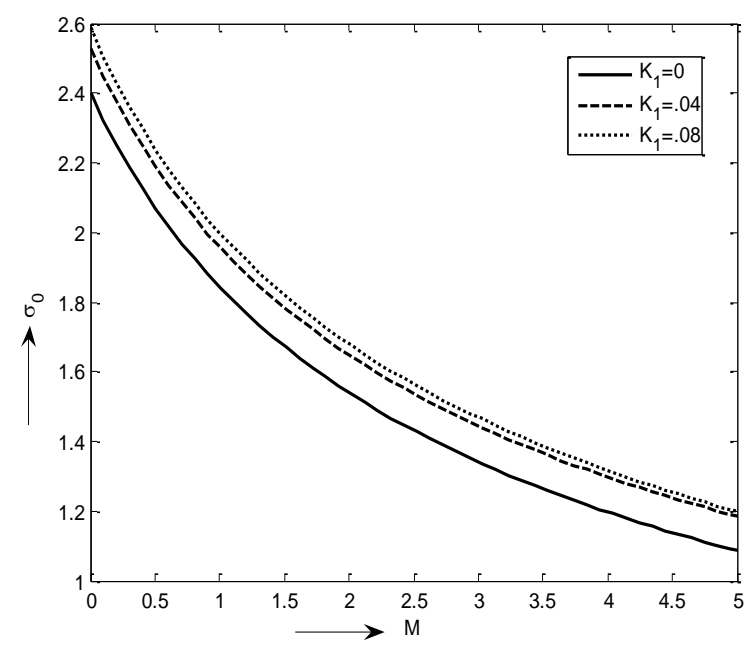

Figure 10: Skin-friction coefficient $\sigma_{0}$ against $M$ for $G_{r}=-5, G_{m}=5, H=3$

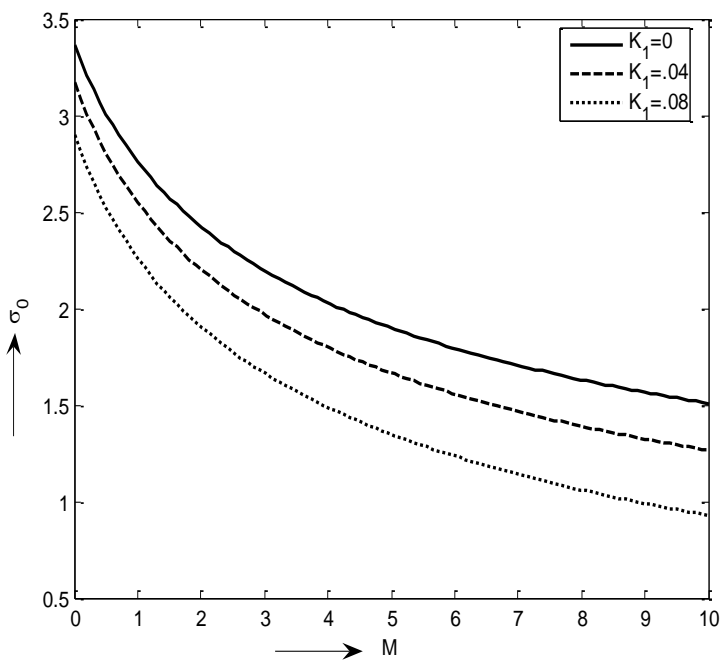

Figure 11: Skin-friction coefficient $\sigma_{0}$ against $M$ for $G_{r}=2, G_{m}=5, H=3$

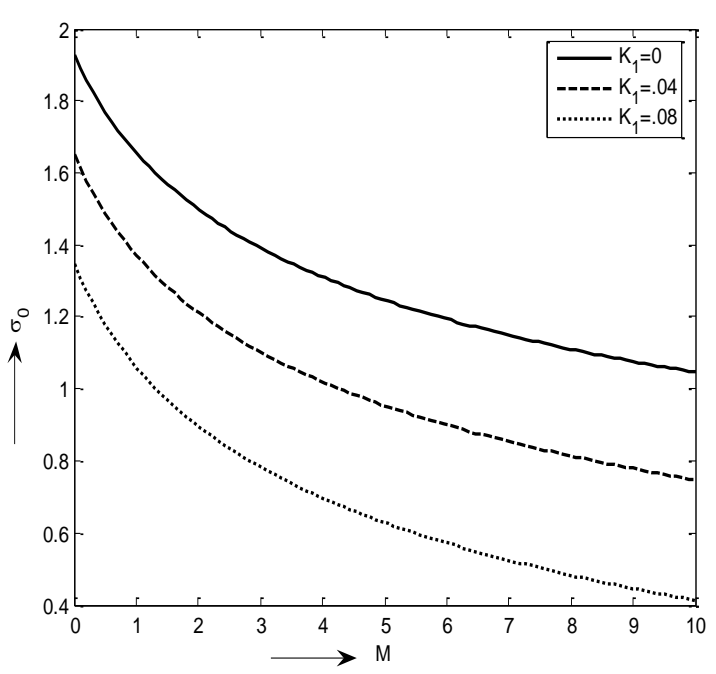

Figure 12: Skin-friction coefficient $\sigma_{0}$ against $M$ for $G_{r}=5, G_{m}=2, H=3$ 


\section{CONCLUSION}

An analysis of mixed convective MHD visco-elastic flow with heat and mass transfer from a vertical plate in presence of ohmic heating and viscous dissipation has been presented for different values of visco-elastic parameter $K_{1}$ in combination of other flow parameters.

The study paves the way for the following conclusions:

* The velocity field is considerably affected at every point of the fluid region by the visco-elastic parameter.

* The velocity field is accelerated during the enhancement of the visco-elastic parameter in comparison with Newtonian fluid.

* The effects of Grashof numbers for heat and mass transfer in the flow field are remarkable.

* The skin friction coefficient against the heat source parameter diminishes on the porous plate with the rising effect of visco-elastic parameter and the heat source parameter as well for externally cooled plate and an opposite trend is observed for externally heated plate.

* The skin-friction coefficient against the magnetic parameter exhibits a decelerating trend with the enhancement of the visco-elastic parameter for externally cooled plate and an opposite trend is noticed for externally heated plate.

* The skin-friction coefficient against the magnetic parameter exhibits a diminishing trend with the growth of the magnetic parameter for both externally cooled and externally heated plates.

* Visco-elasticity has no prominent effect in the thermal and concentration fields.

\section{REFERENCES}

[1] Elbashbeshy, E. M. A. 1997. Heat and Mass transfer along a vertical plate with variable temperature and concentration in the presence of magnetic field. Int. J. Engng. Sci. 34(1997), 515-522.

[2] Hossain, M. A. and Rees, D. A. S. 1999. Combined heat and mass transfer in natural convection flow from a vertical wavy surface. Acta Mechanica. 136(1999), 133-141.

[3] Chien-Hsin-Chen. 2004. Combined heat and mass transfer in MHD free convection from a vertical surface with ohmic heating and viscous dissipation. Int J. Engng. Sci. 42(2004)(7), 699-713.

[4] Acharya, M., Dash, G. C. and Singh, L.P. 2001. Hall Effect with simultaneous thermal and mass diffusion on unsteady hydromagnetic flow near an accelerated vertical plate. Ind. J. Physics. B. 75B(2001)( 1), 68-70.

[5] Aboeldahab, E. M. and Elbarbary, E.M.E. 2001. Hall current effect magneto hydrodynamics free convection flow past a semi infinite vertical plate with mass transfer, Int. J. Engng. Sci. 39(2001), 1641-1652.

[6] Datta, N. and Jana, R. N. 1976. Oscillatory megnatohydrodynamic flow past a flat plate with Hall effects, J. Phys., Soc. Japan. 40(1976), 1469-1475.

[7] Hossain, M. A., Alim, M. A. and Rees, D. A. S. 1999. The effect of radiation on free convection from a porous vertical plate, Int. J. Heat Mass Transfer. 42(1999), $181-191$.

[8] Ghaly, A. Y. and Elbarbary, E. M. E. 2002. Radiation effect on MHD free convection flow of a gas at a stretching surface with a uniform free stream, J. Appl. Math. 2(2002)(2), 93-103.

[9] Muthukumarswamy, R. and Senthil, G. K. 2004. Heat and mass transfer effects on moving vertical plate in the presence of thermal radiation. Theoret, Appl. Mech. 31(2004)(1), 35-46.

[10] Hossain, M. A. 1992. Viscous and Joule heating effects on MHD free convection flow with variable plate temperature. Int. J. Heat Mass Transfer. 35(1992), 3485-3487.

[11]Babu, V. S. H. and Reddy, G. V. R. 2011. Mass transfer effects on MHD mixed convective flow from a vertical surface with ohmic heating and viscous dissipation. Advances in Appl. Sci. Res. 2(2011)(4), 138-146.

[12] Ganesan, P. and Palani, G. 2004,Numerical solution of unsteady MHD flow past a semi-infinite isothermal vertical plate, Proceedings of the 6th ISHMT/ASME Heat and Mass Transfer Conference and 17th National Heat and Mass Transfer Conference, January 5-7, 2004, Kalpakkam, India, pp. 184-187.

[13] Ganesan, P. and Palani, G. (2004). Finite difference analysis of unsteady natural convection MHD flow past an inclined plate with variable surface heat and mass flux. Int. J. of Heat and Mass Transfer, 47(2004)(19-20), 4449-4457.

[14] Aydin, O. and Kaya, A. 2008. Non-Darcian forced convection flow of viscous dissipating fluid over a flat plate embedded in a porous medium. TRANS POROUS MEDIA.73(2008)(2). 173-186.

[15] Aydin, O. and Kaya, A. 2009. MHD mixed convection of a viscous dissipating fluid about permeable vertical flat plate. Appl. Math. Model. 33(2009)(110), 40864096.

[16] Kelly, D., Vajravelu, K. and Andrews, L. 1999. Analysis of heat mass transfer of a visco-elastic, electrically conducting fluid past a continuous stretching sheet. Non-linear Analysis: Theory, Methods and Applications. 36(1999)(6), 767-784.

[17] Subhash, A. M., Joshi, A. and Sonth, R. M. 2001. Heat transfer in MHD visco-elastic fluid flow over a stretching surface, Zeitschrift für angewandte Mathematikund Mechanik. 81(2001)(10), 691-698.

[18] Sonth, R. M., Khan, S. K., Subhash, A. M. and Prasad, K. V. 2002. Heat and mass transfer in a viscoelastic flow over an accelerating surface with heat source/sink and viscous dissipation. Heat and Mass Transfer. 38(2002)(3), 213-220,

[19] Abel, M. S., Siddheshwar, P. G. and Nandeeppanavar, M. M. 2007. Heat transfer in a visco-elastic boundary layer flow over a stretching sheet with viscous dissipation and non-uniform heat source. Int. J. Heat and Mass Transfer. 50(2007)(5-6), 960-966.

[20] Choudhury, R. and Mahanta, M. 2009. Free convective MHD flow of a visco-elastic fluid past an infinite 
vertical channel. Int. J. Appl. Math. 23(2009), 189203.

[21] Choudhury, R. and Dey, D. 2010, Free convective visco-elastic flow with heat and mass transfer through a porous medium with periodic permeability. Int. J. Heat and Mass Transfer (Elsevier). 53(2010), 16661672.

[22] Choudhury, R. and Das, U. 2012, Heat transfer to MHD oscillatory visco-elastic flow in a channel filled with porous medium, Phys. Res..Int. 2012,Ar ID 8795537, 5 pages,.

[23] Bachok, N., Ishak, A. and Pop, I. 2011. MHD flow and heat transfer near the stagnation point on a stretching/shrinking sheet in a micropolar fluid, Magnetohydrodynamics. 47(2011)(3), 237-248,.

[24] Walters, K. 1960. The motion of an elastico-viscous liquid contained between co-axial cylinders (II). Quart. J. Mech. Appl. Math.13 (1960), 444-461.

[25] Walters, K. 1962. The solution of flow problems in the case of materials with memories. J. Mecanique, 1(1962), 473-478.

[26] Cogley, A. C., Vincenty, W. G. and Gilles, S. E. 1968. Differential approximation for radiation transfer in a nongray near equilibrium. AIAAJ. 6(1968), 551-553. 